\title{
The Synthesis, Antimicrobial Activity Studies, and Molecular Property Predictions of Novel Benzothiazole-2-Thione Derivatives
}

\author{
Barkın Berk $^{1 \star}$, Yesim Altas Tahirovic², Emre Fatih Bülbül1', Sevde Nur Biltekin ${ }^{1}$ \\ ${ }^{1}$ İstanbul Medipol University, School of Pharmacy, Kavacık Mah. Ekinciler Cad. No.19 Kavacık Kavşağı, TR-34810, \\ Beykoz, İstanbul, Turkey. \\ 2 Emory University, Chemistry Department, 1515 Dickey Drive, Atlanta, GA 30322, USA.
}

\begin{abstract}
Benzothiazoles and 2-mercaptobenzothiazoles are important classes of bioactive organic scaffolds possessing antibacterial, antifungal, antitubercular, antiinflammatory, antidiabetic, and antimalarial properties. In recent years, prediction of druglikeness, molecular, absorption, distribution, metabolism, and excretion (ADME) properties using in silico techniques has become a standard procedure for the evaluation of molecules in terms of their potential clinical use. In this study, compounds structured 6-benzoyl-3-substitutedmethylbenzo[d]thiazole-2( $3 H$ )-thione were synthesized using the Mannich reaction starting from 2-mercaptobenzothiazole. The antibacterial and antifungal activities of these compounds were determined against Staphylococcus aureus, Escherichia coli, Enterococcus faecalis, Pseudomonas aeruginosa, Candida albicans, Candida krusei, and Candida parapisilosis using a broth microdilution method. An additional analysis was undertaken using the in silico technique to predict the drug-likeness, molecular, and ADME properties of these molecules. Among all the compounds, respectively, Compounds 1-4 and 6-11 exhibited good minimum inhibition concentration values against Staphylococcus aureus and Candida species with promising predicted properties.
\end{abstract}

Keywords: Benzothiazole-2-thione, ADME, antifungal, Candida albicans, molecular properties, in silico

\section{INTRODUCTION}

Today, the misuse of antibiotics has become an important global concern in terms of causing antimicrobial resistance. Fluoroquinolone resistance of Escherichia coli is very widespread and this treatment is now ineffective in more

*Corresponding author, Tel.; +90-216-6815364 Fax: +90-212-5317555.

E-mail address: bberk@medipol.edu.tr (Barkın Berk). 
than half of the patients. Furthermore, it is estimated that people with methicillin-resistant Staphylococcus aureus (MRSA) are 64\% more likely to die of this infection compared to people with a non-resistant form. The situation is similar for resistance in Klebsiella pneumoniae, Enterobacteriaceae and HIV infections, gonorrhea, tuberculosis, malaria, and influenza. Clearly, development of new active compounds for the antimicrobial resistance of bacteria, fungi, viruses, and parasites is a priority ${ }^{1}$.

Benzothiazole (BTA) analogs are one of the most versatile classes of compounds which are a common and integral feature of a variety of natural products and pharmaceutical agents. BTA derivatives have attracted continuing interest due to their diverse biological activities including anticancer, antimicrobial, anticonvulsant, antiviral, antitubercular, antimalarial, antihelminthic, analgesic, antiinflammatory, antidiabetic, and fungicidal activities ${ }^{2}$.

Previous studies have shown that compounds derived from the $2^{\text {nd }}, 5^{\text {th }}$, and $6^{\text {th }}$ positions of benzothiazole and 2-mercaptobenzothiazole structures by various functional groups are effective antimicrobial and antifungal agents. In addition, substitution of electron withdrawing groups such as amino, nitro, trifluoromethyl, and halogens especially at the $6^{\text {th }}$ position enhances the antimicrobial and antifungal activities ${ }^{3-13}$. In their literature review, Keri et.al. ${ }^{2}$ and Azam and Suresh $^{14}$ found that the pharmacological activity of these systems has been widely investigated and found efficient.

In a very early study, Halasa and Smith suggested that the benzothiazole-2-thiol ring system was in a tautomeric form with benzothiazole-2-thione (Figure 1) and Michael/Mannich reactions could easily be performed to produce N-substituted benzothiazole-2-thione compounds with excellent yields ${ }^{15}$.

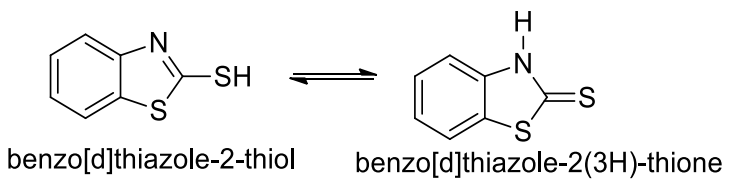

Figure 1. Tautomeric forms of the benzothiazole-2-thiol ring system

Mannich bases have been reported to exhibit antifungal and antimicrobial activities when connected to various ring systems ${ }^{16}$. Furthermore utilizing the antibacterial $^{17-20}$, antispasmodic ${ }^{21}$, and antitubercular activities ${ }^{22}$ of the benzothiazole-2-thione ring system, Varma et al. synthesized a series of N-substituted5-(hydrogen/chloro)benzothiazole-2-thiones and found that the compounds

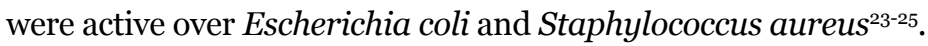

Electronic behavior of benzothiazole-2-thione is similar to that of the benzothia- 
zol-2-one ring. Studies on the latter have shown that acylation of this ring system at the $6^{\text {th }}$ position can be achieved either by Friedel-Craft conditions or through the reaction of carboxylic and polyphosphoric acids ${ }^{26}$. Considering these findings, addition of a moderately electron withdrawing group such as benzoyl to the $6^{\text {th }}$ position of the benzothiazole-2-thione ring system and performing a Mannich reaction with the ring nitrogen can result in products with antimicrobial or antifungal activities.

In this study, we synthesized 6-benzoyl-3-substitutedmethylbenzo[d]thiazole2(3H)-thione derivatives. We also determined the antibacterial and antifungal activities of the compounds against Staphylococcus aureus, Escherichia coli, Enterococcus faecalis, Pseudomonas aeruginosa, Candida albicans, Candida krusei, and Candida parapisilosis using a broth microdilution method. Furthermore, their absorption, distribution, metabolism, and excretion (ADME), druglikeness, and molecular properties were predicted by in silico techniques.

\section{METHODOLOGY}

\section{Chemistry}

All the chemicals were purchased from Aldrich Chemical Co. (Steinheim, Germany). Melting points were determined with a Mettler-Toledo FP62 capillary melting point apparatus (Columbus, $\mathrm{OH}$, USA). IR spectra ( $\mathrm{KBr}$ ) were recorded on a PerkinElmer Spectrum One FT-IR spectrometer (Waltham, MA, USA) and 1H-NMR spectra were obtained by Bruker DPX-400, 400 MHz High Performance Digital FT-NMR. All the chemical shift values were recorded as $\delta$ (ppm). Mass spectra were recorded using an Agilent 1100 series LC/APCI/MS 1946 G spectrometer in the negative ionization mode. The purity of the compounds was checked by thin-layer chromatography on silica gel-coated aluminum sheets (Merck, 1.005554, silica gel HF254-361, Type 60, o.25 mm; Darmstadt, Germany). Elemental analyses were performed with a Leco CHNS 932 analyzer (Leco Corp., MI, USA) and found to be within $\pm 0.4 \%$ of the theoretical values for C, $\mathrm{H}$, and $\mathrm{N}$.

\section{General synthesis}

A solution of 2-mercaptobenzothiazole (40 mmol) in $150 \mathrm{~mL}$ of polyphosphoric acid was reacted portion wise with benzoic acid (50 mmol) under mechanical stirring and further heated to $130^{\circ} \mathrm{C}$ for $12 \mathrm{hr}$. After cooling, the reaction mixture was poured onto ice-water. The precipitated 6-benzoylbenzo[d]thiazole-2 $(3 H)$ thione was filtered, washed with ice-cold water, and recrystallized from ethanol. Then, 6-benzoylbenzo[d]thiazole-2(3H)-thione (0.5 mol) was suspended in 20 $\mathrm{ml}$ of ethanol followed by the addition of first $7.5 \mathrm{ml}$ of $37 \%$ formalin to this 
suspension and then an appropriate secondary amine (0.05 mol). The reaction mixture was stirred at room temperature for $4 \mathrm{hr}$ with occasional warming on a water bath. After cooling and filtering, the final products were collected from the reaction vessel, washed with cold ether, and recrystallized from the ethanolacetone mixture as solids (Scheme 1).

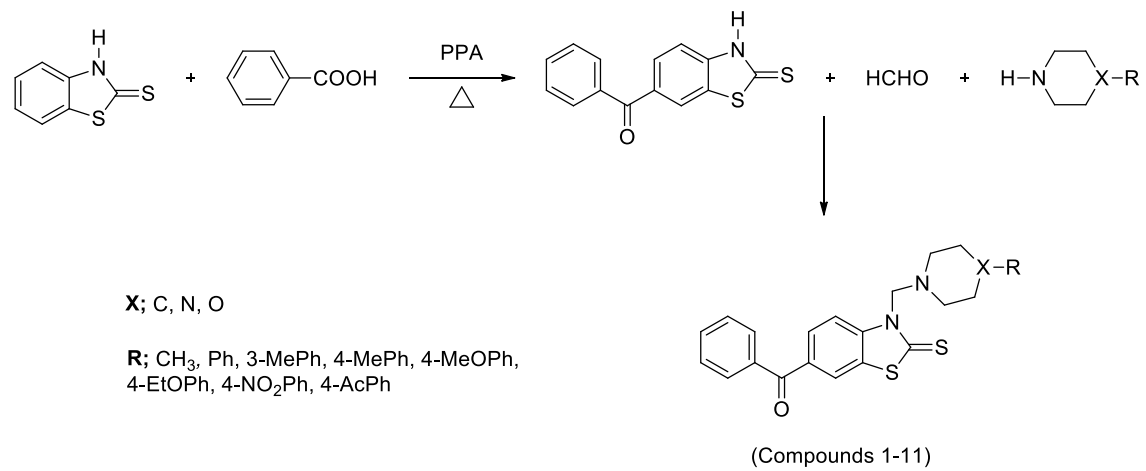

Scheme 1. Synthetic pathway followed for the preparation of 3-substitutedmethyl-6benzoylbenzo[d] thiazole-2(3H)-thione derivatives (Compounds 1-11)

\section{6-Benzoyl-3-(piperidin-1-ylmethyl)benzo[d]thiazole-2(3H)-thione (Compound 1)}

Yield 60\%, M.p.: $213{ }^{\circ} \mathrm{C}$, white solid. IR (KBr) $\bar{v}_{\max }\left(\mathrm{cm}^{-1}\right): 3000(\mathrm{CH}$, aromatic), 2795 (CH, aliphatic), 1680 (C=O). ${ }^{1} \mathrm{H}-\mathrm{NMR}$ (400 MHz, DMSO-d6, $\delta$ ): 1.5- 1.6 (m, 6H, Pip H), 2.45 (t, J=7.1O Hz, 4H, Pip H), 4.15 (s, 2H, - $\mathrm{CH}_{2}-$ ), 7.43-7.59 (m, $3 \mathrm{H}, \mathrm{Ar} \mathrm{H}$ ), 7.72 (d, $J=8.2 \mathrm{~Hz} 2 \mathrm{H}, \mathrm{Ar} \mathrm{H}$ ), 7.80 (d, $J=9.5 \mathrm{~Hz}, 2 \mathrm{H}, \mathrm{Ar} \mathrm{H}), 8.50$ (s, $1 \mathrm{H}, \mathrm{ArH}) \mathrm{ppm}$. MS $368.1\left(\mathrm{M}^{+}\right)$. Anal. calcd for $\mathrm{C}_{20} \mathrm{H}_{20} \mathrm{~N}_{2} \mathrm{OS}_{2}: \mathrm{C}, 65.18 ; \mathrm{H}, 5.47 ; \mathrm{N}$, 7.60. Found: C, 65.16; H, 5.44; N, 7.62.

\section{6-Benzoyl-3-(morpholin-4-ylmethyl)benzo[d]thiazole-2(3H)-thione (Compound 2)}

Yield 50\%, M.p.: $186{ }^{\circ} \mathrm{C}$, white solid. IR (KBr) $\bar{v}_{\max }\left(\mathrm{cm}^{-1}\right): 3005(\mathrm{CH}$, aromatic), 2805 (CH, aliphatic), $1685(\mathrm{C}=\mathrm{O}) .{ }^{1} \mathrm{H}-\mathrm{NMR}$ (400 MHz, DMSO-d6, 8): 2.50 (t, $J=7.00 \mathrm{~Hz}, 4 \mathrm{H}, \mathrm{Mor}$ ), 3.65 (t, $J=7.00 \mathrm{~Hz}, 4 \mathrm{H}, \mathrm{Mor}$ ), 4.14 (s, 2H, $-\mathrm{CH}_{2}-$ ), 7.45$7.61(\mathrm{~m}, 3 \mathrm{H}, \mathrm{Ar} \mathrm{H}), 7.74$ (d, $J=8.2 \mathrm{~Hz}, 2 \mathrm{H}, \mathrm{Ar} \mathrm{H}), 7.82$ (d, $J=9.5 \mathrm{~Hz}, 2 \mathrm{H}, \mathrm{Ar} \mathrm{H})$, $8.52(\mathrm{~s}, 1 \mathrm{H}, \mathrm{ArH}) \mathrm{ppm}$. MS $370.0\left(\mathrm{M}^{+}\right)$. Anal. calcd for $\mathrm{C}_{19} \mathrm{H}_{18} \mathrm{~N}_{2} \mathrm{O}_{2} \mathrm{~S}_{2}: \mathrm{C}, 61.60 ; \mathrm{H}$, 4.90; N, 7.56. Found: C, 61.58; H, 4.88; N, 7.55.

\section{6-Benzoyl-3-(piperazin-1-ylmethyl)benzo[d]thiazole-2(3H)-thione (Compound 3)}

Yield 70\%, M.p.: $208^{\circ} \mathrm{C}$, white solid. IR (KBr) $\bar{v}_{\text {max }}\left(\mathrm{cm}^{-1}\right): 3305$ (NH, Pip.), 3008 (CH, aromatic), 2810 (CH, aliphatic), $1689(\mathrm{C}=\mathrm{O}) .{ }^{1} \mathrm{H}-\mathrm{NMR}$ (400 MHz, DMSO- 
d6, 8): 1.98 (s, 1H, Ppz NH), 2.37 (t, 4H, Ppz H), 3.42 (t, 4H, Ppz H), 4.15 (s, 2H, $\left.-\mathrm{CH}_{2}{ }^{-}\right), 7.43-7.59$ (m, 3H, Ar H), 7.72 (d, $\left.J=8.2 \mathrm{~Hz}, 2 \mathrm{H}, \mathrm{Ar} \mathrm{H}\right), 7.80$ (d, $J=9.5 \mathrm{~Hz}$, $2 \mathrm{H}, \mathrm{Ar} \mathrm{H}), 8.50(\mathrm{~s}, 1 \mathrm{H}, \mathrm{Ar} \mathrm{H})$ ppm. MS $369.1\left(\mathrm{M}^{+}\right)$. Anal. calcd for $\mathrm{C}_{19} \mathrm{H}_{19} \mathrm{~N}_{3} \mathrm{OS}_{2}$ : C, 61.76; H, 5.18; N, 11.37. Found: C, 61.73; H, 5.19; N, 11.40.

\section{6-Benzoyl-3-((4-methylpiperazin-1-yl)methyl)benzo[d]thiazole- 2(3H)-thione (Compound 4)}

Yield 78\%, M.p.: $220{ }^{\circ} \mathrm{C}$, white solid. IR $(\mathrm{KBr}) \bar{v}_{\text {max }}\left(\mathrm{cm}^{-1}\right): 3008(\mathrm{CH}$, aromatic), 2820 (CH, aliphatic), $1650(\mathrm{C}=\mathrm{O}) .{ }^{1} \mathrm{H}-\mathrm{NMR}$ (400 MHz, DMSO-d6, 8): 2.26 (s, $3 \mathrm{H}, \mathrm{Ppz}_{-} \mathrm{CH}_{3}$ ), 2.35 (s, 8H, Ppz H), 4.18 (s, 2H, - $\mathrm{CH}_{2}-$ ), 7.43-7.59 (m, 3H, Ar H), 7.70 (d, $J=8.2 \mathrm{~Hz}, 2 \mathrm{H}, \mathrm{Ar} \mathrm{H}$ ), 7.79 (d, $J=9.5 \mathrm{~Hz}, 2 \mathrm{H}, \operatorname{Ar} \mathrm{H}$ ), 8.54 (s, 1H, ArH) ppm. MS 383.1 $\left(\mathrm{M}^{+}\right)$. Anal. calcd for $\mathrm{C}_{20} \mathrm{H}_{21} \mathrm{~N}_{3} \mathrm{OS}_{2}: \mathrm{C}, 62.63 ; \mathrm{H}, 5.52 ; \mathrm{N}, 10.96$. Found: C, 62.65; H, 5.56; N, 10.93 .

\section{6-Benzoyl-3-((4-phenylpiperazin-1-yl)methyl)benzo[d]thiazole- 2(3H)-thione (Compound 5)}

Yield 80\%, M.p.: $242{ }^{\circ} \mathrm{C}$, white solid. IR (KBr) $\bar{v}_{\max }(\mathrm{cm}-1): 3050(\mathrm{CH}$, aromatic), 2930 (CH, aliphatic), 1655 (C=O). ${ }^{1} \mathrm{H}-\mathrm{NMR}$ (400 MHz, DMSO-d6, 8): 2.49 (t, 4H, Ppz H), 2.65 (t, 4H, Ppz H), 4.15 (s, 2H, - $\mathrm{CH}_{2}$-), 6.78-7.1 (m, 5H, Ppz-Phe), 7.43-7.59 (m, 3H, ArH), 7.72 (d, $J=8.2 \mathrm{~Hz}, 2 \mathrm{H}, \mathrm{ArH}), 7.80$ (d, $J=9.5 \mathrm{~Hz}, 2 \mathrm{H}$, ArH), 8.50 (s, $1 \mathrm{H}, \mathrm{ArH})$ ppm. MS 445.1 $\left(\mathrm{M}^{+}\right)$. Anal. calcd for $\mathrm{C}_{25} \mathrm{H}_{23} \mathrm{~N}_{3} \mathrm{OS}_{2}: \mathrm{C}$, 67.39; H, 5.20; N, 9.43. Found: C, 67.41; H, 5.23; N, 9.39.

\section{6-Benzoyl-3-((4-(3-methylphenyl)piperazin-1-yl)methyl)benzo[d] thiazole-2(3H)-thione (Compound 6)}

Yield 70\%, M.p.: $190{ }^{\circ} \mathrm{C}$, white solid. IR $(\mathrm{KBr}) \bar{v}_{\text {max }}\left(\mathrm{cm}^{-1}\right): 3050(\mathrm{CH}$, aromatic), 2940 (CH, aliphatic), 1649 (C=O). ${ }^{1} \mathrm{H}-\mathrm{NMR}$ (400 MHz, DMSO-d6, 8): 2.35 (s, $3 \mathrm{H}, \mathrm{Phe}-\mathrm{CH}_{3}$ ), 2.48 (t, 4H, Ppz H), 3.44 (t, 4H, Ppz H), 4.15 (s, 2H, - $\mathrm{CH}_{2}-$ ), 6.607.1 (m, 4H, Ppz-Phe), 7.43-7.59 (m, 3H, ArH), 7.72 (d, J=8.2 Hz, 2H, ArH), 7.80 (d, $J=9.5 \mathrm{~Hz}, 2 \mathrm{H}, \mathrm{ArH}), 8.50(\mathrm{~s}, 1 \mathrm{H}, \mathrm{ArH}) \mathrm{ppm}$. MS $459.1\left(\mathrm{M}^{+}\right)$. Anal. calcd for $\mathrm{C}_{26} \mathrm{H}_{25} \mathrm{~N}_{3} \mathrm{OS}_{2}: \mathrm{C}, 67.94 ; \mathrm{H}, 5.48 ; \mathrm{N}, 9.14$. Found: C, 67.98; H, 5.44; N, 9.10.

\section{6-Benzoyl-3-((4-(4-methylphenyl)piperazin-1-yl)methyl)benzo[d] thiazole-2(3H)-thione (Compound 7 )}

Yield 74\%, M.p.: $215{ }^{\circ} \mathrm{C}$, white solid. IR $(\mathrm{KBr}) \bar{v}_{\max }\left(\mathrm{cm}^{-1}\right): 3049(\mathrm{CH}$, aromatic), 2930 (CH, aliphatic), 1648 (C=O). ${ }^{1} \mathrm{H}-\mathrm{NMR}$ (400 MHz, DMSO-d6, 8): 2.34 (s, $3 \mathrm{H}, \mathrm{Phe}_{-} \mathrm{CH}_{3}$ ), 2.48 (t, 4H, Ppz H), 3.44 (t, 4H, Ppz H), 4.15 (s, 2H, - $\mathrm{CH}_{2}-$ ), 6.64 (d, J=9.o Hz , 2H, Ppz-Phe), 7.05 (d, J=9.o Hz, 2H, Ppz-Phe), 7.43-7.59 (m, $3 \mathrm{H}, \mathrm{ArH}$ ), 7.72 (d, $J=8.2 \mathrm{~Hz}, 2 \mathrm{H}, \mathrm{ArH}$ ), 7.80 (d, $J=9.5 \mathrm{~Hz}, 2 \mathrm{H}, \mathrm{ArH}), 8.50$ (s, $1 \mathrm{H}, \mathrm{ArH}) \mathrm{ppm}$. MS 459.1 ( $\left.\mathrm{M}^{+}\right)$. Anal. calcd for $\mathrm{C}_{26} \mathrm{H}_{25} \mathrm{~N}_{3} \mathrm{OS}_{2}: \mathrm{C}, 67.94 ; \mathrm{H}, 5.48 ; \mathrm{N}$, 9.14. Found: C, 67.90; H, 5.45; N, 9.12. 


\section{6-Benzoyl-3-((4-(4-methoxyphenyl)piperazin-1-yl)methyl)benzo[d] thiazole-2(3H)-thione (Compound 8)}

Yield 70\%, M.p.: $252^{\circ} \mathrm{C}$, white solid. IR $(\mathrm{KBr}) \overline{\mathrm{v}}_{\max }\left(\mathrm{cm}^{-1}\right): 3055(\mathrm{CH}$, aromatic), 2924 (CH, aliphatic), 1649 (C=O). ${ }^{1} \mathrm{H}-\mathrm{NMR}$ (400 MHz, DMSO-d6, 8): 2.48 (t, $4 \mathrm{H}, \mathrm{Ppz} \mathrm{H}), 3.44$ (t, $4 \mathrm{H}, \mathrm{Ppz} \mathrm{H}), 3.84\left(\mathrm{~s}, 3 \mathrm{H},-\mathrm{OCH}_{3}\right), 4.13\left(\mathrm{~s}, 2 \mathrm{H},-\mathrm{CH}_{2}-\right), 6.65^{-}$ 6.81 (m, 4H, Ppz-Phe), 7.43-7.59 (m, 3H, ArH), 7.72 (d, $J=8.2 \mathrm{~Hz}, 2 \mathrm{H}, \mathrm{ArH})$, $7.80(\mathrm{~d}, J=9.5 \mathrm{~Hz}, 2 \mathrm{H}, \mathrm{ArH}), 8.50(\mathrm{~s}, 1 \mathrm{H}, \mathrm{ArH})$, ppm. MS 475.1 ( $\left.\mathrm{M}^{+}\right)$. Anal. calcd for $\mathrm{C}_{26} \mathrm{H}_{25} \mathrm{~N}_{3} \mathrm{O}_{2} \mathrm{~S}_{2}: \mathrm{C}, 65.66 ; \mathrm{H}, 5.30 ; \mathrm{N}, 8.83$. Found: C, 65.63; H, 5.33; N, 8.80.

\section{6-Benzoyl-3-((4-(4-ethoxyphenyl)piperazin-1-yl)methyl)benzo[d] thiazole-2(3H)-thione (Compound 9)}

Yield 85\%, M.p.: $194{ }^{\circ} \mathrm{C}$, white solid. IR $(\mathrm{KBr}) \bar{v}_{\max }\left(\mathrm{cm}^{-1}\right): 3040(\mathrm{CH}$, aromatic), 2920 (CH, aliphatic), 1652 (C=O). ${ }^{1} \mathrm{H}-\mathrm{NMR}$ (400 MHz, DMSO-d6, 8): 1.32 (t, $\left.J=8.0 \mathrm{~Hz}, 3 \mathrm{H},-\mathrm{CH}_{3}\right), 2.48(\mathrm{t}, 4 \mathrm{H}, \mathrm{Ppz} \mathrm{H}), 3.44(\mathrm{t}, 4 \mathrm{H}, \mathrm{Ppz} \mathrm{H}), 4.09(\mathrm{q}, 2 \mathrm{H}$, $-\mathrm{OCH}_{3}$ ), $4.13\left(\mathrm{~s}, 2 \mathrm{H},-\mathrm{CH}_{2}-\right.$ ), $6.65-6.81$ (m, 4H, Ppz-Phe), 7.43-7.59 (m, $3 \mathrm{H}$, $\operatorname{ArH}$ ), 7.72 (d, $J=8.2 \mathrm{~Hz}, 2 \mathrm{H}, \operatorname{ArH}), 7.8 \mathrm{o}(\mathrm{d}, J=9.5 \mathrm{~Hz}, 2 \mathrm{H}, \operatorname{ArH}), 8.50(\mathrm{~s}, 1 \mathrm{H}$, ArH), ppm. MS 489.2 (M+). Anal. calcd for $\mathrm{C}_{27} \mathrm{H}_{27} \mathrm{~N}_{3} \mathrm{O}_{2} \mathrm{~S}_{2}: \mathrm{C}, 66.23 ; \mathrm{H}, 5.56 ; \mathrm{N}$, 8.58. Found: C, 66.27; H, 5.6o; N, 8.61.

\section{6-Benzoyl-3-((4-(4-nitrophenyl)piperazin-1-yl)methyl)benzo[d]thi- azole-2(3H)-thione (Compound 10)}

Yield 55\%, M.p.: $234{ }^{\circ} \mathrm{C}$, white-reddish solid. IR (KBr) $\bar{v}_{\max }\left(\mathrm{cm}^{-1}\right): 3020(\mathrm{CH}$, aromatic), 2918 (CH, aliphatic), $1649(\mathrm{C}=\mathrm{O}) .{ }^{1} \mathrm{H}-\mathrm{NMR}$ (400 MHz, DMSO-d6,

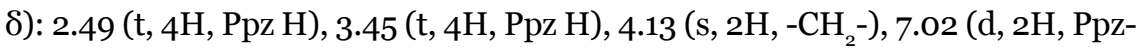
Phe), 7.43-7.59 (m, 3H, ArH), 7.72 (d, $J=8.2 \mathrm{~Hz}, 2 \mathrm{H}, \mathrm{ArH}), 7.8 \mathrm{o}$ (d, $J=9.5 \mathrm{~Hz}$, 2H, ArH), 8.50 (s, 1H, ArH), 8.70 (d, 2H, Ppz-Phe) ppm. MS 490.1 (M+). Anal. calcd for $\mathrm{C}_{25} \mathrm{H}_{22} \mathrm{~N}_{4} \mathrm{O}_{3} \mathrm{~S}_{2}: \mathrm{C}, 61.2 \mathrm{O} ; \mathrm{H}, 4.52 ; \mathrm{N}, 11.42$. Found: C, 61.18; H, 4.49; N, 11.39.

\section{6-Benzoyl-3-((4-(4-acetylphenyl)piperazin-1-yl)methyl)benzo[d]thi- azole-2(3H)-thione (Compound 11)}

Yield 64\%, M.p.: $263{ }^{\circ} \mathrm{C}$, white. IR ( $\left.\mathrm{KBr}\right) \bar{v}_{\max }\left(\mathrm{cm}^{-1}\right): 3024(\mathrm{CH}$, aromatic), 2926 (CH, aliphatic), 1655 (C=O). ${ }^{1} \mathrm{H}-\mathrm{NMR}$ (400 MHz, DMSO-d6, $\delta$ ): 2.48 (t, 4H, Ppz $\mathrm{H}), 2.5 \mathrm{O}\left(\mathrm{s}, 3 \mathrm{H}, \mathrm{CH}_{3}\right.$ ), 3.44 (t, $\left.4 \mathrm{H}, \mathrm{Ppz} \mathrm{H}\right), 4.13\left(\mathrm{~s}, 2 \mathrm{H},-\mathrm{CH}_{2}-\right), 6.85$ (d, 2H, PpzPhe), 7.43-7.59 (m, 3H, ArH), 7.72 (d, $J=8.2$ Hz, 2H, ArH), 7.77 (d, 2H, PpzPhe), 7.80 (d, $J=9.5 \mathrm{~Hz}, 2 \mathrm{H}, \mathrm{ArH}$ ), 8.50 (s, $1 \mathrm{H}, \mathrm{ArH}$ ), ppm. MS $487.1\left(\mathrm{M}^{+}\right)$. Anal. calcd for $\mathrm{C}_{27} \mathrm{H}_{25} \mathrm{~N}_{3} \mathrm{O}_{2} \mathrm{~S}_{2}:$ C, 66.50; H, 5.17; N, 8.62. Found: C, 66.53; H, 5.15; N, 8.61 . 


\section{Microbiological Screening}

The following test microorganisms were obtained from LGC Standards GmbH (Wesel, Germany): Staphylococcus aureus ATCC 25923, Escherichia coli ATCC 25922, Enterococcus faecalis ATCC 29212, Pseudomonas aeruginosa ATCC 27853, Candida albicans ATCC 60193, Candida krusei ATCC 28870, and Candida parapisilosis ATCC 90018. All the synthesized compounds were dissolved in dimethyl sulfoxide (DMSO) to prepare a stock solution at $10 \mathrm{mg} / \mathrm{mL}$.

\section{Broth microdilution method}

The minimal inhibition concentration (MIC) values $(\mu \mathrm{g} / \mathrm{mL}$ ) for the organisms were determined using the methods recommended by the Clinical and Laboratory Standards Institute (CLSI) guidelines ${ }^{27-28}$. The antimicrobial effects of the substances against all the microorganisms were quantitatively tested in broth media using double dilution. The antibacterial and antifungal assays were performed in a Mueller Hinton broth (Difco) at pH 7.3 and a buffered yeast nitrogen base (Difco) at pH 7.0, respectively. MIC was defined as the lowest concentration with no bacterial or fungal growth. Carbenicillin $(10 \mu \mathrm{g} / \mathrm{mL})$ and fluconazole $(10 \mu \mathrm{g} /$ $\mathrm{mL}$ ) were prepared as stocks, then diluted in a range from 10 to $0.5 \mu \mathrm{g} / \mathrm{mL}$ using DMSO, and tested as standard antibacterial and antifungal drugs, respectively. For all the compounds, the tested dilutions ranged from 128 to $0.5 \mu \mathrm{g} / \mathrm{mL}$ using DMSO as the solvent. The control samples prepared with the amounts of DMSO used in the dilutions did not show any inhibitory activity under these conditions.

Table 1: Antimicrobial activity of compounds using microdilution (MIC, $\mu \mathrm{g} / \mathrm{mL}$ )

\begin{tabular}{|c|c|c|c|c|c|c|c|}
\hline Compound & 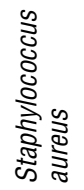 & 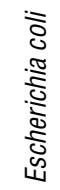 & 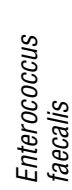 & 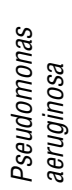 & 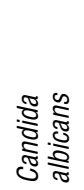 & 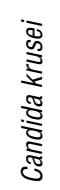 & 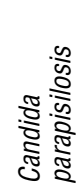 \\
\hline 1 & 8 & 128 & 128 & 256 & 32 & 64 & 64 \\
\hline 2 & 8 & 256 & 128 & 256 & 32 & 64 & 64 \\
\hline 3 & 16 & 128 & 128 & 128 & 32 & 64 & 64 \\
\hline 4 & 4 & 64 & 256 & 128 & 64 & 128 & 64 \\
\hline 5 & 64 & 64 & 256 & 256 & 64 & 32 & 32 \\
\hline 6 & 64 & 128 & 256 & 256 & 64 & 32 & 16 \\
\hline 7 & 128 & 128 & 256 & 128 & 64 & 16 & 8 \\
\hline 8 & 128 & 64 & 128 & 128 & 16 & 8 & 4 \\
\hline 9 & 64 & 64 & 128 & 128 & 16 & 8 & 4 \\
\hline 10 & 32 & 64 & 256 & 256 & 8 & 16 & 16 \\
\hline 11 & 64 & 64 & 128 & 256 & 8 & 8 & 4 \\
\hline Carbenicillin & 4 & 8 & 32 & 32 & & & \\
\hline Fluconazole & & & & & 0.5 & 64 & 4 \\
\hline
\end{tabular}




\section{Prediction of drug-likeness, molecular and ADME properties}

All the molecules were prepared in $3 \mathrm{D}$ using the LigPrep module of Maestro (Schrodinger Inc.). The ADME properties (46 molecular descriptors) were determined using the QikProp program (Schrödinger 2015-3) in the normal mode. QikProp generates physically relevant descriptors, which are then used to perform ADME predictions. An overall ADME-compliance score, the drug-likeness parameter (indicated by \#stars), was used to assess the pharmacokinetic profiles of the compounds. The \#stars parameter (ranging from o to 5) indicates the number of property descriptors computed by QikProp that fall outside the optimum range of values for $95 \%$ of known drugs. The following descriptors were predicted: Central nervous system (CNS) activity (from -2 for inactive to +2 for active); octanol/water partition coefficient, $\log \mathrm{Po} / \mathrm{w}$ ( -2.0 to 6.5 ); $\mathrm{IC}_{50}$ value for the block of HERG $\mathrm{K}^{+}$channels, $\log H E R G$ (concern $<-5$ ); Caco-2 cell membrane permeability in $\mathrm{nm} \mathrm{s}^{-1}$, PCaco (: < 5 low to > 100 high); logarithm of the predicted blood/brain barrier partition coefficient, $\log \mathrm{B} / \mathrm{B}$ (-3.0 to 1.0); apparent Madin-Darby canine kidney cell permeability (PMDCK) that mimic the blood-brain barrier for non-active transport in $\mathrm{nm} \mathrm{s}^{-1}$, PMDCK $(<25$ poor to $>500$ great); skin permeability, $\log K_{\mathrm{p}}$ (-8.0 to -1.0 ); logarithm of binding constant to human serum albumin, $\log K_{\mathrm{HSA}}$ (-1.5 to 1.2); qualitative human oral absorption (HOA) (1: low, 2: medium, 3: high); percent of HOA (>80\%: high, $<25 \%$ : poor) (Table 2).

Table 2: The calculated drug-likeness, molecular properties and ADME predictions for Compounds 1-11 using QikProp

\begin{tabular}{|c|c|c|c|c|c|c|c|c|c|c|c|}
\hline $\begin{array}{l}\text { 릏 } \\
\text { 을 } \\
\text { 헝 }\end{array}$ & 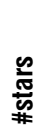 & $\sum_{0}^{\infty}$ & $\begin{array}{l}\text { 총 } \\
\text { 음 }\end{array}$ & $\begin{array}{l}\text { ్ㅗ } \\
\text { 岌 } \\
\text { 吾 }\end{array}$ & $\begin{array}{l}\stackrel{0}{0} \\
\text { ঠूँ }\end{array}$ & $\begin{array}{l}\boldsymbol{0} \\
\stackrel{0}{0}\end{array}$ & $\begin{array}{l}\text { 䓌 } \\
\text { 玄 }\end{array}$ & 흠 & 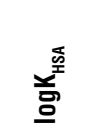 & ઠิ & 옹 \\
\hline 1 & 0 & 2 & 3.729 & -6.521 & 781.726 & 0.463 & 1698.396 & -3.241 & 0.187 & 3 & 100 \\
\hline 2 & 0 & 2 & 2.617 & -6.047 & 820.341 & 0.513 & 1731.346 & -3.207 & -0.395 & 3 & 94.425 \\
\hline 3 & 0 & 2 & 2.325 & -7.064 & 105.042 & 0.59 & 205.004 & -5.823 & 0.042 & 3 & 76.735 \\
\hline 4 & 0 & 2 & 2.388 & -7.238 & 175.945 & 0.793 & 329.151 & -5.362 & -0.248 & 3 & 81.116 \\
\hline 5 & 0 & 1 & 4.822 & -7.772 & 804.78 & 0.437 & 1673.878 & -2.564 & 0.528 & 3 & 100 \\
\hline 6 & 0 & 1 & 4.933 & -7.443 & 756.618 & 0.412 & 1611.668 & -2.871 & 0.621 & 3 & 100 \\
\hline 7 & 0 & 1 & 5.244 & -7.751 & 824.606 & 0.433 & 1750.35 & -2.729 & 0.738 & 3 & 96.89 \\
\hline 8 & 0 & 1 & 4.75 & -7.67 & 725.135 & 0.297 & 1376.039 & -2.734 & 0.469 & 3 & 100 \\
\hline 9 & 0 & 1 & 5.204 & -7.908 & 723.683 & 0.211 & 1373.061 & -2.651 & 0.63 & 3 & 95.636 \\
\hline 10 & 1 & 0 & 4.082 & -7.722 & 82.714 & -0.794 & 143.123 & -4.598 & 0.458 & 3 & 85.166 \\
\hline 11 & 0 & 1 & 4.023 & -7.692 & 231.066 & -0.288 & 399.738 & -3.742 & 0.249 & 3 & 92.808 \\
\hline
\end{tabular}




\section{RESULTS}

Eleven 6-benzoyl-3-substitutedmethylbenzo[d]thiazole-2(3H)-thione derivatives were successfully synthesized by the Mannich method giving yields between 50 and $85 \%$. In the IR spectra, all the compounds had a strong $\mathrm{C}=\mathrm{O}$ stretching band for the benzoyl group at $1648-1689 \mathrm{~cm}^{-1}$. The ${ }^{1} \mathrm{H}-\mathrm{NMR}$ spectra of the compounds showed that the protons belonging to the 6-benzoylbenzo[d] thiazole-2 $(3 H)$-thione ring system exhibited similar properties to those reported by previous studies ${ }^{15}{ }^{29}$. All the other protons were observed according to the expected chemical shift and integral values. The molecular ion peaks $\left(\mathrm{M}^{+}\right)$of the compounds were examined under electron ionization and confirmed the molecular weights of the compounds. The MIC values of Compounds 1-11 were evaluated for their antibacterial and antifungal activities using carbenicillin and fluconazole as a standard for the microorganisms. For practical purposes, the MIC values $\leq 16 \mu \mathrm{g} / \mathrm{mL}$ were considered to be active for the evaluation of the results. None of the synthesized compounds was found to be active against Escherichia coli, Escherichia. faecalis, and Pseudomonas. aeruginosa as much as the standard carbenicillin. According to the results of Staphylococcus aureus, Compounds 1-4 showed an antibacterial activity comparable to the same standard. Similarly, for Candida albicans, Compounds 8-11 showed some activity but performed worse than fluconazole. Compounds 7-11 had a strong activity against Candida krusei resulting in similar inhibition concentrations of 8 to $16 \mu \mathrm{g} / \mathrm{mL}$, which was better than fluconazole with the inhibition concentration of $64 \mu \mathrm{g} /$ mL. In the broth dilution experiments, Compounds 6, 7, and 10 were found to have an MIC of 8 to $16 \mu \mathrm{g} / \mathrm{mL}$, which had a moderate activity against Candida parapisilosis. Another significant result was that Compounds 8, 9, and 11 had MIC values of $4 \mu \mathrm{g} / \mathrm{mL}$ presenting the same anti-fungal activity as fluconazole. Interestingly, compounds with either small substituents at the $4^{\text {th }}$ position or non-substituted 6-Benzoyl-3-(piperidinyl/piperazinyl/morpholin-4-ylmethyl) benzo[d] thiazole-2(3H)-thione derivatives were active against Staphylococcus aureus. On the contrary the aromatic functional groups containing complex substituents were more active against Candida species.

In this study, the drug-likeness, molecular and ADME properties of all compounds were promising presenting a drug-like/lead-like profile according to their \#stars rankings. The \#stars rankings were o for all the compounds except Compound 10 (1). The combinations of HOA values being mostly around 3, \%HOA values ranging from 76.73 to $100 \%$, all PCaco values being high and log Kp values varying between -2.54 and -5.82 indicate that these compounds can be effectively used in both oral and topical preparations. The $\log K_{\mathrm{HSA}}$ values varying between -0.35 and +0.45 indicates that the proposed molecules can freely 
circulate and easily traverse cell membranes without binding to human serum albumin. For determining the cardiac toxicity of drugs in the early stages of drug discovery, HERG $\mathrm{K}^{+}$channel blockage activity is very important ${ }^{30}$. The $\log \mathrm{H}-$ ERG values of the compounds predicted with the in silico method were between -6.04 and -7.90 ; thus, they can be considered safe for human use. The blood/ brain partition coefficient $(\log B \mathrm{~B}), \mathrm{PMDCK}$, and $\log \mathrm{Po} / \mathrm{w}$ values are useful to determine the penetration capacity of a compound from blood-brain barrier. The values predicted for these parameters of the synthesized compounds were within the ranges defined for $95 \%$ of drugs. Moreover, the predicted CNS value of the compounds was between $\mathrm{o}$ and 2 indicating medium to high activity.

The results of the activity analyses showed that the synthesized 6-benzoyl3 -substitutedmethylbenzo[d]thiazole-2(3H)-thione derivatives could be considered as potential effective antifungal agents against Candida species. Further studies are necessary to confirm and validate the predictive results based on actual experiments.

\section{REFERENCES}

1. Antimicrobial Resistance, Fact Sheet. World Health Organisation: September,2016.

2. Keri, R. S.; Patil, M. R.; Patil, S. A.; Budagupi, S., A Comprehensive Review in Current Developments of Benzothiazole-Based Molecules in Medicinal Chemistry. Eur. J. Med. Chem. 2015, 89, 207-251.

3. Franchini, C.; Muraglia, M.; Corbo, F.; Florio, M. A.; Di Mola, A.; Rosato, A.; Matucci, R.; Nesi, M.; van Bambeke, F.; Vitali, C., Synthesis and Biological Evaluation of 2-Mercapto-1,3-Benzothiazole Derivatives with Potential Antimicrobial Activity. Arch. Pharm. 2009, 342 (10), 605-613.

4. Seenaiah, D.; Reddy, P. R.; Reddy, G. M.; Padmaja, A.; Padmavathi, V.; Siva krishna, N., Synthesis, Antimicrobial and Cytotoxic Activities of Pyrimidinyl Benzoxazole, Benzothiazole and Benzimidazole. Eur. J. Med. Chem. 2014, 77, 1-7.

5. Padalkar, V. S.; Borse, B. N.; Gupta, V. D.; Phatangare, K. R.; Patil, V. S.; Umape, P. G.; Sekar, N., Synthesis and Antimicrobial Activity of Novel 2-Substituted Benzimidazole, Benzoxazole and Benzothiazole Derivatives. Arabian J. Chem. 2016, 9 (Suppl._2), S1125-S1130.

6. Soni, B.; Ranawat, M. S.; Sharma, R.; Bhandari, A.; Sharma, S., Synthesis and Evaluation of Some New Benzothiazole Derivatives as Potential Antimicrobial Agents. Eur. J. Med. Chem. 2010, 45 (7), 2938-2942.

7. Singh, M. K.; Tilak, R.; Nath, G.; Awasthi, S. K.; Agarwal, A., Design, Synthesis and Antimicrobial Activity of Novel Benzothiazole Analogs. Eur. J. Med. Chem. 2o13, 63, 635-644.

8. Al-Talib, M.; Al-Soud, Y. A.; Abussaud, M.; Khshashneh, S., Synthesis and Biological Evaluation of New Benzothiazoles as Antimicrobial Agents. Arabian J. Chem. 2o16, 9 (Suppl._1), S926S930.

9. Gabr, M. T.; El-Gohary, N. S.; El-Bendary, E. R.; El-Kerdawy, M. M.; Ni, N.; Shaaban, M. I., Synthesis, Antimicrobial, Antiquorum-Sensing and Cytotoxic Activities of New Series of Benzothiazole Derivatives. Chin. Chem. Lett. 2015, 26 (12), 1522-1528. 
10. Gill, R. K.; Rawal, R. K.; Bariwal, J., Recent Advances in the Chemistry and Biology of Benzothiazoles. Arch. Pharm. 2015, 348 (3), 155-178.

11. Chugunova, E.; Boga, C.; Sazykin, I.; Cino, S.; Micheletti, G.; Mazzanti, A.; Sazykina, M.; Burilov, A.; Khmelevtsova, L.; Kostina, N., Synthesis and Antimicrobial Activity of Novel Structural Hybrids of Benzofuroxan and Benzothiazole Derivatives. Eur. J. Med. Chem. 2o15, 93, 349-359.

12. Kuchta, T.; Bujdakova, H.; Sidoova, E., Inhibition of Yeast-Mycelium Transformation by 2-Alkylthio-6-Amino- and 2-Alkylthio-6-Formamidobenzothiazoles and Their in Vitro Antifungal Activity. Folia Microbiol. 1989, 34 (6), 504-10.

13. Sidoova, E.; Loos, D.; Bujdakova, H.; Kallova, J., New Anticandidous 2-Alkylthio-6-Aminobenzothiazoles. Molecules 1997, 2 (2), 36-42.

14. Azam, M. A.; Suresh, B., Biological Activities of 2-Mercaptobenzothiazole Derivatives: A Review. Sci. Pharm. 2012, 80 (4), 789-823.

15. Halasa, A. F.; Smith, G. E. P., Michael and Mannich Reactions with Benzothiazole-2-Thiol. The J. Org. Chem. 1971, 36 (5), 636-641.

16. Bala, S.; Sharma, N.; Kajal, A.; Kamboj, S.; Saini, V., Mannich Bases: An Important Pharmacophore in Present Scenario. Int. J. Med. Chem. 2014, 191072/1-191072/16.

17. Moys, A.; Bloekinger, G.; Schwartz, E., Chemical and Microbiological Properties of 2-Mercaptobenzothiazole with Regard to Treatment of Skin Tuberculosis and Some Infectious Dermatoses. Cesko-Slov. Dermatol. 1964, 39 (4), 269-74.

18. Chatterjee, M. G.; Ranganathan, S. K.; Saxena, B. B. L.; Sengupta, S. R., Rapid Assay for Fungicides. Defence Sci. J. 1961, 11 (3), 170-5.

19. Kuznetsova, E. A.; Zhuravlev, S. V.; Stepanova, T. N.; Solov'ev, V. N.; Zueva, V. S., Synthesis and Antibacterial Activity of Some Derivatives of 2-Mercaptobenzothiazole. Khim.-Farm. Zh. 1967, 1(2), 7-10.

20. Cossey, H. D.; Gartside, R. N.; Stephens, F. F., The Antimicrobial Activity of Benzothiazole Basic Ethers and Related Compounds. Some Structure-Activity Relations. Arzneim.-Forsch. 1966, 16 (1), 33-40.

21. Zapadnyuk, V. I., The Dependence of Anticonvulsion Activity and Toxicity of Thiohydantoin and Rhodanine Derivatives on the Chemical Structure. Farm. Zh. (Kiev) 1962, 17 (No. 1), 36-41.

22. Moys, A.; Schwartz, E.; Bloeckinger, G., 2-Mercaptobenzothiazole and Its Derivatives in the Treatment of Skin Tuberculosis. Bratisl. Lek. Listy 1963, 43-II (6), 325-32.

23. Varma, R. S., Potential Biologically Active Agents. Ii. Synthesis of Substituted 3-(Aminomethyl)Benzothiazoline-2-Thiones. J. Prakt. Chem. 1972, 314 (5-6), 955-7.

24. Varma, R. S.; Nobles, W. L., New Compounds: N-Substituted Benzothiazoline-2-Thiones. $J$ Pharm Sci 1969, 58 (4), 497-8.

25. Varma, R. S.; Nobles, W. L., Substituted 3-Aminomethylbenzoxazoline-2-Thiones as Potential Antibacterial Agents. J. Pharm. Sci. 1972, 61 (1), 112-13.

26. Yous, S.; Poupaert, J. H.; Lesieur, I.; Depreux, P.; Lesieur, D., Alcl3-Dmf Reagent in the Friedel-Crafts Reaction. Application to the Acylation Reaction of 2(3h)-Benzothiazolones. J. Org. Chem. 1994, 59 (6), 1574-6.

27. Performance Standards for Antimicrobial Susceptibility Testing Twenty-Third Informational Supplement. CLSI Document. M10o-S20. 2013. 
28. Methods for Dilution Antimicrobial Susceptibility Tests for Bacteria That Grow Aerobically; Approved Standard-Ninth Edition, CLSI Document: Mo7-A9. 2012.

29. Deligeorgiev, T. G.; Kaloyanova, S. S.; Lesev, N. Y.; Vaquero, J. J., An Environmentally Benign Procedure for the Synthesis of Substituted 2-Thiobenzothiazoles, 2-Thiobenzoxazoles, 2-Thiobenzimidazoles, and 1,3-Oxazolopyridine-2-Thiols. Monatsh. Chem. 2011, 142 (9), 895899.

30. Aronov, A. M., Predictive in Silico Modeling

(Received o8 April 2017; Accepted 23 April 2017) 